\title{
Amniotic fluid fluorescence polarisation values for assessing fetal lung maturation
}

\author{
M LEGGE, HC POTTER \\ From the Perinatal Biochemistry and Pathology Services, Christchurch Women's Hospital, \\ Christchurch, New Zealand
}

SUMMARY The fluorescence probe 1,6, diphenyl-1,3,5-hexatriene was used to determine the microviscosity of third trimester amniotic fluid samples utilising an Aminco Bowman spectrofluorometer fitted with a polariser prism. The results were related to the lecithin sphingomyelin $(\mathrm{L}: \mathrm{S})$ ratio. A fluorescence polarisation value of 0.360 corresponded to an $\mathrm{L}: \mathrm{S}$ ratio of 2.0 in uncomplicated and diabetic pregnancies. Pregnancies complicated by Rhesus disease gave significantly different fluorescence polarisation values when compared with the uncomplicated pregnancies.

The use of the lecithin to sphingomyelin ratio ( $\mathrm{L}: \mathrm{S}$ ratio) has proved invaluable in the prenatal assessment of fetal lung maturation, and has become an established technique in many laboratories. When the ratio of lecithin to sphingomyelin exceeds $2 \cdot 0$ there is minimal risk of the infant developing respiratory distress. Other tests for assessing fetal lung maturation have been introduced such as total phospholipids, ${ }^{1}$ free fatty acids, ${ }^{2}$ surface tension measurements ${ }^{3}$ and the stability of amniotic foam. ${ }^{4}$

In 1971, Shinitzky et al. ${ }^{5}$ described a technique whereby the microviscosity of lipid systems could be determined using fluorescent probes. This was based on the principle that a lipid soluble fluorescent dye will preferentially absorb into phospholipid dispersions and is dependent upon the phospholipid acyl chain length, degree of saturation, and the concentration of lecithin to sphingomyelin. Subsequent work by Cogan et al. ${ }^{6}$ and Shinitzky and Barenholz ${ }^{7}$ demonstrated that the fluorescent probe 1,6, diphenyl-1,3,5-hexatriene (DPH) was of value in determining the microviscosity of phospholipid and phospholipid-cholesterol vesicles (liposomes). As amniotic fluid phospholipids are thought to exist as liposomes, ${ }^{89}$ and an empirical relation exists between liposomes, viscosity and surface tension, Shinitzky et $a l .{ }^{10}$ investigated the use of DPH to assess fetal lung maturity. An inverse relation was demonstrated between increasing $L: S$ ratio and the microviscosity of amniotic fluid. These findings led to the production of the Eliscant fetal lung maturity analyser, a dedicated instrument costing approximately SUS 14000 .

Accepted for publication 18 March 1981
In this report we have investigated the use of the DPH technique for assessing fetal lung maturation using a standard Aminco Bowman spectrofluorometer, fitted with a polarisation prism.

\section{Material and methods}

SAMPLES

Amniotic fluid, free from meconium, was collected by transabdominal amniocentesis for fetal maturity assessment. In this series a total of 70 amniotic fluid samples, free from blood were tested. Of these, 50 were from uncomplicated pregnancies; 13 were from pregnancies complicated by Rhesus disease; and seven were from pregnancies complicated by maternal diabetes mellitus. In addition to these a further 10 moderately blood-stained amniotic fluid samples were tested. All samples were centrifuged immediately after collection and had an $L: S$ ratio performed and quantified as described by Legge. ${ }^{11}$

\section{REAGENTS}

The fluorescent probe 1,6-diphenyl-1,3,5-hexatriene was purchased from Koch-Light Laboratories, Colnbrook, UK. Tetrahydrofuran (Fissons, AR), was used without further purification. The stock DPH solution was prepared by dissolving $5 \mathrm{mg}$ of DPH in $10 \mathrm{ml}$ of tetrahydrofuran. This may be stored in sealed ampoules at $4^{\circ} \mathrm{C}$ until required. A working solution of DPH is prepared by injecting $50 \mu \mathrm{l}$ of stock DPH into $100 \mathrm{ml}$ of vigorously stirred phosphate-buffered saline pH 7.4 at $25^{\circ} \mathrm{C}$. A fresh DPH dispersion needs to be prepared daily.

METHOD

Five hundred microlitres of centrifuged amniotic 
fluid is mixed with $2 \mathrm{ml}$ of the DPH dispersion and incubated at $37^{\circ} \mathrm{C}$ for $30 \mathrm{~min}$. After incubation the sample is allowed to cool to $24^{\circ} \mathrm{C} \pm 1^{\circ} \mathrm{C}$. The fluorescence polarisation is then read using a fluorometer with polarised excited light at $360 \mathrm{~nm}$ and emission $426 \mathrm{~nm}$. It is not necessary to have the emission wavelength polarised. In this series an Aminco Bowman spectrofluorometer was used to which a Glan prism polariser (Aminco Bowman cat no A546-62140) had been fitted to the sample compartment. This can be easily performed by laboratory personnel.

Two adjustments are made to the polarisation prism. The first orientates the prism to pass only light polarised parallel to the direction of the excited light (designated $I_{11}$ ) and the second orientates the prism to pass only light perpendicular to the direction of polarisation of the excited light (designated $I_{1}$ ). The degree of fluorescence polarisation was then calculated using the formula shown below:

$$
\text { Fluorescence polarisation }(P)=\frac{I_{11}-I_{1}}{I_{11}+I_{1}}
$$

\section{Results}

An inverse relation was demonstrated between the $\mathrm{L}: \mathrm{S}$ ratio and fluorescence polarisation (Figure). A Bravais-Pearson correlation coefficient of -0.763 with a linear regression line of $y=0.4185-0.00295 x$ and a standard error of estimate of $y$ on $x$ of 0.0259 was determined for the uncomplicated pregnancy group. Using the calculated regression line a fluorescence polarisation value of $\mathbf{0 . 3 6 0}$ corresponded to an $\mathrm{L}: \mathrm{S}$ ratio of $\mathbf{2 \cdot 0 .}$

Fluorescence polarisation values from the pregnancies complicated by Rhesus disease, maternal diabetes mellitus and blood stained amniotic fluids were compared with uncomplicated pregnancies using the $\mathrm{F}$ test and $t$ test for unpaired data. The Rhesus disease group demonstrated a significant difference in the fluorescence polarisation values, $F=2.69(p<0.05)$. The $L: S$ ratio in this group corresponded to a fluorescence polarisation value of $0 \cdot 330$. The maternal diabetes mellitus fluorescence polarisation values were not significantly different using both the $\mathrm{F}$ and $t$ tests; $\mathrm{F}=2 \cdot 0, t=1 \cdot 1(\mathrm{p}>0.05)$. Similarly, no significant difference in fluorescence polarisation values was demonstrated with moderately blood-stained amniotic fluids using both the $\mathrm{F}$ and $t$ tests, $\mathrm{F}=1.23 ; t=0.886(\mathrm{p}>0.05)$.

No infant born with a fluorescence polarisation value of 0.360 or less, in this series, developed respiratory distress syndrome.

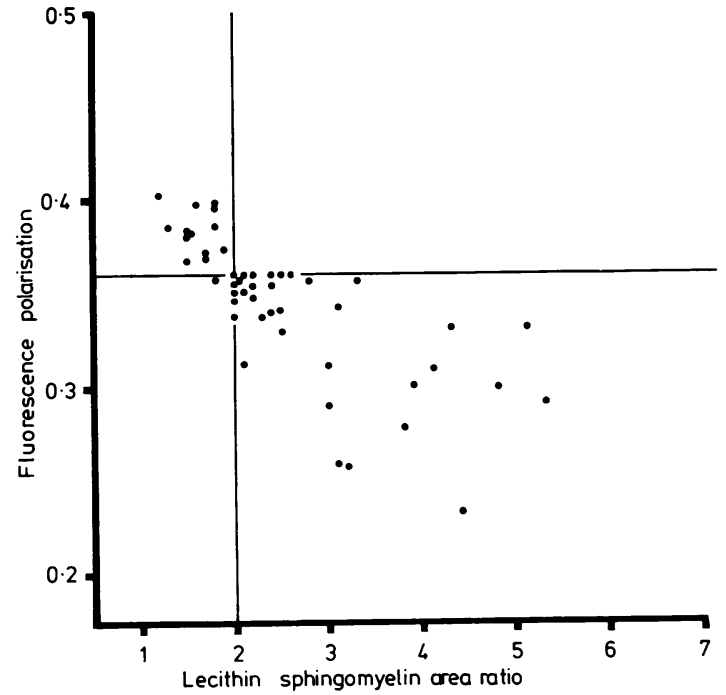

Comparison of amniotic fluid fluorescence polarisation values and the lecithin sphingomyelin area ratio in 50 uncomplicated pregnancies.

\section{Discussion}

A method has been described which is well suited for the rapid assessment of fetal lung maturation, correlating well with fetal outcome and the $L: S$ ratio. The close association of fluorescence polarisation and $\mathrm{L}: \mathrm{S}$ ratio as described by Shinitzky et al. ${ }^{10}$ and other workers ${ }^{1213}$ using dedicated instrumentation has been confirmed using a standard fluorometer fitted with a polariser. This should make the technique readily available to more laboratories who would not normally have the resources to purchase a dedicated analyser to determine fetal lung maturation. We can see no reason why this technique cannot be used on the simpler fluorometers providing suitable light source and filters are used.

As microviscosity relates to the total phospholipid, this technique is not a lecithin-specific method. However, as the major phospholipids present in third trimester are dipalmitoyl lecithin and phosphatidylglycerol, ${ }^{14}$ it follows that they would be the major contributors to the microviscosity of the amniotic fluid. Other phospholipids remain relatively constant.

At present no explanation can be given for the significant difference between fluorescence polarisation values for Rhesus disease pregnancies and uncomplicated pregnancies. This is being investigated further. Further investigations are also being conducted on the significance of amniotic fluid microviscosity and the relation of fetal phospholipid metabolism in complicated pregnancies. 
This work was supported, in part, by a grant from the Medical Research Council of New Zealand.

\section{References}

${ }^{1}$ Gudson JP, Waiti BM. A colorimetric method for amniotic fluid phospholipids and their relation to the respiratory distress syndrome. Am J Obstet Gynecol 1972; 112:62-71.

${ }^{2}$ Legge M. The assessment of fetal lung maturation using amniotic fluid palmitic acid quantitation. NZ J Med Lab Technol 1980;34:79-82.

${ }^{3}$ Muller-Tyle E, Lampert S, Steinbereithner K, Benzer H. Surface properties of the amniotic fluid in normal pregnancy. Am J Obstet Gynecol 1975;122:295-300.

${ }^{4}$ Clements JA, Platzker ACG, Tierney DF, et al. Assessment of the risk of the respiratory distress syndrome by a rapid test for surfactant in amniotic fluid. $N$ Engl J Med 1972;286:1077-81.

${ }^{5}$ Shinitzky M, Dianoux AC, Gitler C, Weber G. Microviscosity and order in the hydrocarbon region of micelles and membranes determined with fluorescent probes. 1. Synthetic micelles. Biochemistry 1971;10: 2106-13.

${ }^{6}$ Cogan U, Shinitzky M, Weber G, Nishida T. Microviscosity and order in the hydrocarbon region of phospholipid and phospholipid-cholesterol dispersions determined with fluorescent probes. Biochemistry $1973 ; 12: 521-8$.

${ }^{7}$ Shinitzky M, Barenholz Y. Dynamics of the hydrocarbon layer in liposomes of lecithin and sphingomyelin con- taining dicetylphosphate. $J$ Biol Chem 1974;249:2652-7.

${ }^{8}$ Blumenfeld TA, Cheskin HS, Shinitzky M. Microviscosity of amniotic fluid phospholipids, and its importance in determining fetal lung maturity. Clin Chem 1979;25:64-7.

${ }^{9}$ Frosolono MF, Charms BL, Pawlowski R. Isolation, characterisation and surface chemistry of a surfaceactive fraction from dog lung. J Lipid Res 1970;11: 439-57.

${ }^{10}$ Shinitzky M, Goldfisher A, Bruck A, et al. A new method for assessment of fetal lung maturity. $\mathrm{Br} J$ Obstet Gynaecol 1976;83:838-44.

${ }^{11}$ Legge M. Lecithin to sphingomyelin ratio-a comparison of two methods of assessment. NZ J Med Lab Technol 1976;30:84-5.

${ }^{12}$ Stark FI, Blumenfeld TA, George JD, Freda VJ, James LS. Amniotic fluid microviscosity determined by fluorescence polarization: Methodology and relation to gestational age. Pediatrics 1979;63:213-8.

${ }^{13}$ Stark RI, Blumenfeld TA, Cheslain HS, Dyrenfurth I, James LS. Amniotic fluid fluorescence polarisation value as a predictor of respiratory distress syndrome. J Pediatr 1980;96:301-4.

${ }^{14}$ Obladen M. Factors influencing surfactant composition in the newborn infant. Eur J Pediatr 1978;128:129-43.

Requests for reprints to: Dr M Legge, Department of Pathology, Christchurch Women's Hospital, Christchurch, New Zealand. 